\title{
Effect of very high magnetic field on the optical properties of firefly light emitter oxyluciferin
}

Weihang Zhou ${ }^{1}$, Daisuke Nakamura ${ }^{1}$, Yu Wang ${ }^{1,2}$, Toshimitsu Mochizuki ${ }^{1,3}$, Hidefumi Akiyama $^{1}$, and Shojiro Takeyama ${ }^{1 *}$

${ }^{1}$ Institute for Solid State Physics, University of Tokyo, 5-1-5, Kashiwanoha, Kashiwa, Chiba 277-8581, Japan

${ }^{2}$ State Key Laboratory of Molecular Developmental Biology, Institute of Genetics and Developmental Biology, Chinese Academy of Sciences, China

${ }^{3}$ Fukushima Renewable Energy Institute, National Institute of Advanced Industrial Science and Technology, 2-2-9 Machiike-dai, Koriyama, Fukushima 963-0215, Japan

E-mail: takeyama@issp.u-tokyo.ac.jp

KEYWORDS: firefly, bioluminescence, oxyluciferin, magnetic field, photoluminescence

Magnetic field effect on enzymatic reactions is under intensive study in the past decades. Recently, it was reported that firefly bioluminescence was suppressed and red-shifted significantly when exposed to external magnetic field. However in this work, by means of selective excitation, we confirmed that emission properties of firefly light emitter "oxyluciferin" are completely immune to external magnetic field of up to $53 \mathrm{~T}$. These findings pose strong contrast to existing relevant results. Potential reasons for the discrepancies found and the underlying physics towards the understanding of firefly bioluminescence were discussed. 


\section{Introduction}

Magnetic field effect on enzymatic reactions is a subject of extensive study in the past decades[1-3], as human beings and other living creatures are exposed to all kinds of magnetic fields more and more frequently. Understanding and controlling enzymatic reactions are not only fundamentally interesting, but also practically important. Recently, it was reported that the emission of light by a living creature, i.e. bioluminescence, is affected by external magnetic field significantly[4]. Iwasaka and his co-workers demonstrated, using living fireflies, that firefly bioluminescence was suppressed in intensity and red-shifted for $10 \mathrm{~nm}$ when exposed to a $14 \mathrm{~T}$ magnetic field. As an ideal "cold light" system with extremely high quantum yield of up to $41.0 \pm 7.4 \%$ [5], firefly bioluminescence lies in the center of biochemistry research[6-19]. The revealing of magnetic field effects on firefly bioluminescence therefore represents significant progress in this field. However, as living fireflies were used in their work, there are concerns regarding the accuracy of the spectroscopy measurement. Furthermore, effects of much higher magnetic fields on firefly bioluminescence, as well as the mechanism for magnetic field induced spectral changes, remain unclear so far. Further work is needed to clarify these issues.

In this work, we report high-field magneto-optical study on the emission properties of firefly light emitter oxyluciferin, aiming to clarify the effects of high magnetic field on enzymatic reactions and the underlying mechanism. Unlike in the work by Iwasaka and his co-workers, we employed in vitro luciferin-luciferase reaction, instead of living fireflies, to increase the stability and reproducibility in the experiment and thus the experimental accuracy could be improved greatly.

As a result of the efforts devoted, it is nowadays clear, that firefly bioluminescence results from the oxidation process of the substrate molecule luciferin, with the help of the enzyme luciferase $[5,7,20-30]$. As illustrated in Fig. 1, firefly bioluminescence is known to be a multi-step process. In the first step, the substrate luciferin is oxidized by molecular 
oxygen at the luciferase active site, with adenosine-5'-triphosphate (ATP) and magnesium ion as cofactors, and generates a high energy intermediate product dioxetanone. Dioxetanone, whose decomposition mechanism is under intensive study [31-33], is unstable. It decomposes and generates the final product oxyluciferin in an electronically excited state. Photon emission, i.e. bioluminescence, happens when the electronically excited oxyluciferin relaxes to its ground state. From these reaction steps, one could see clearly, that oxyluciferin is the real light emitter in firefly bioluminescence. Therefore, photoluminescence (PL) from oxyluciferin is essentially a close analogue to firefly bioluminescence. Moreover, compared with bioluminescence, PL from oxyluciferin gives continuous and stable emission as long as external pumping is on. It could thus facilitate a much more reliable optical measurement.

\section{Experimental}

On the other hand, to simulate the micro-environment of firefly bioluminescence, the oxyluciferin molecules used in this work were generated directly from the luciferin-luciferase reaction. The enzyme we used is Luciola cruciata (Lcr) luciferase recombinated from Escherichaia coli. The activity and bioluminescent reaction catalyzed by this enzyme have been confirmed to be the same as the wild type luciferase in our previous work [34]. GTA buffer solution ( $0.15 \mathrm{M}$, containing $0.05 \mathrm{M}$ 3,3-dimethylglutaric acid, $\quad 0.05 \quad \mathrm{M} \quad$ 2-amino-2-hydroxymethyl-1,3-protanediol, $\quad$ and $\quad 0.05 \quad \mathrm{M}$ 2-amino-2-methyl-1,3-propanediol) was used to dilute the reactants to the expected concentrations. The concentration of the D-luciferin was estimated to be $4.0 \times 10^{-8} \mathrm{M}$. The Lcr luciferase solution of the estimated concentration of $8.0 \times 10^{-6} \mathrm{M}$ was prepared by dissolving the luciferase powder with the GTA buffer solution containing $10 \%$ glycerol. The $\mathrm{pH}$ of the reaction solution was $\sim 6.6$. The concentration of the luciferase was made much higher than that of the substrate luciferin to ensure that all luciferin molecules can be consumed in the luciferin-luciferase reaction. The in situ PL measurement was performed 
right after the luciferin-luciferase reaction completed. All measurements were finished within one hour after the reaction, during which the reaction product was stable [35].

For magneto-optical measurement, the pulsed high magnetic field was generated by a home-made wire-wound solenoid coil with maximum field of $\sim 53 \mathrm{~T}$ and pulse duration time of $\sim 40 \mathrm{~ms}$ [36-37]. The excitation laser beam and the photoluminescence signal from oxyluciferin were both guided by optical fibers. All magneto-photoluminescence spectra were taken on the top of the pulsed magnetic field with detector gate opening time of $\sim 5$ ms, during which the field variation is maintained within $4 \%$.

\section{Results and discussion}

Typical bioluminescence spectrum of the $L c r$ fireflies $(300 \mathrm{~K}, \mathrm{pH} \sim 6.6)$ is shown in Fig. 2(a). A broad peak, centering around $2.1 \mathrm{eV}$, can be clearly observed. However, the spectrum is highly asymmetric in its lineshape, implying strongly that it consists of several components that probably correspond to different species of the oxyluciferin. This issue has been clarified in our previous work using quantitative spectroscopy [5, 34]. In Refs. 5

\& 34, our quantitative spectroscopy measurements reveal that firefly bioluminescence spectrum can be systematically decomposed into three Gaussian components, which locate in the green, orange and red spectral regions, respectively. To compare with these existing results, we also carried out curve fittings using three Gaussian profiles, the same as we did in Refs. 5 \& 34. As expected, the bioluminescence spectrum is well reproduced by three Gaussian peaks locating at $\sim 2.2, \sim 2.0$ and $\sim 1.88 \mathrm{eV}$, respectively, as shown in Fig. 2(a).

To find out the effects of high magnetic field on firefly bioluminescence and the underlying mechanism, one efficient way is to study how these three Gaussian components respond to the external magnetic field. Fortunately, it is nowadays clear that these three Gaussian components originate from different species of the light emitter oxyluciferin and can be selectively excited [10, 20-22, 35]. Typical in situ PL spectra of the oxyluciferin in complex with luciferase in the reaction mixture are shown in Fig. 2(b). The fluorescence 
spectra with excitation at $430 \mathrm{~nm}(\sim 2.88 \mathrm{eV}$, green curve $)$ and $532 \mathrm{~nm}(\sim 2.33 \mathrm{eV}$, pink curve) peak at $\sim 2.2 \mathrm{eV}$ and $\sim 1.97 \mathrm{eV}$, respectively. Compared with the Gaussian fittings of the bioluminescence spectrum, it can be seen clearly, that the photoluminescence with $430 \mathrm{~nm}$ excitation (green curve) resembles closely the green component in the bioluminescent spectrum (Peak "G", Fig. 2(a)). On the other hand, the PL spectrum with excitation at $532 \mathrm{~nm}$ (pink curve) shows an obvious low energy tail. By comparison, it can be identified to be an analogue of the orange (Peak "O") and red (Peak "R") components in the bioluminescence shown in Fig. 2(a).

Now we turn our attention to the effects induced by high magnetic field on the emission properties of the oxyluciferin. Fig. 3 shows the typical PL spectra of oxyluciferin under high magnetic fields with near-resonance laser excitation at $406 \mathrm{~nm}$, which is designed to be an analogue to the green component of firefly bioluminescence. Compared with the PL spectrum resonantly excited using a halogen lamp as shown in Fig. 2(b), the spectral width significantly reduces, as a result of the small spectral width $(\sim 0.2 \mathrm{~nm})$ of the excitation laser beam. Upon application of high magnetic field, however, it is surprising to find that the PL spectra coincide with each other even up to the highest field of $53 \mathrm{~T}$.

However, it should be pointed out, that spectral changes that could be detected depend on the resolution of the measurement system. Spectral changes should be estimated based on the measurement resolution. Taking into account the final size of the pixel of the CCD detector and dispersion of the spectrometer, the resolution limit is evaluated to be \pm 0.54 meV for the peak shift and $\sim 1.4 \%$ for the peak intensity. Compared with the high magnetic field data, no spectral changes above these values of the estimated resolution could be found. Thus, it could be concluded that the emission spectra are completely immune to the external high magnetic field.

Similar phenomenon was also found for the photoluminescence with laser excitation at $532 \mathrm{~nm}$ (pink curve, Fig.2 (b)), which is designed to be an analogue to the orange and red 
Gaussian components in firefly bioluminescence. As shown in Fig. 4, when subjected to high magnetic fields up to $52.7 \mathrm{~T}$, negligible changes were found in the emission spectra. The spectral changes again fall within the experimental errors.

While we have confirmed that emission properties of firefly light emitter oxyluciferin do not change with external high magnetic field of even up to $53 \mathrm{~T}$, we now turn our attention to the comparison with Iwasaka's work. In the work by Iwasaka and his co-workers, the bioluminescence spectrum was reported to be red-shifted for $\sim 10 \mathrm{~nm}$ and the emission intensity decreased when the living fireflies were exposed to a $14 \mathrm{~T}$ magnetic field [4]. However in our work, negligible spectral changes could be detected even up to the highest field of $53 \mathrm{~T}$. As oxyluciferin is the real light emitter in firefly bioluminescence and thus PL from oxyluciferin is essentially an analogue to the bioluminescence, the findings in our work are in severe discrepancies with Iwasaka's observation. As living fireflies were used in their work, experimental accuracy could be one possible reason for the observed spectral changes. However, if the measurements in Iwasaka's work are accurate enough, several other factors could be responsible. The first one is that the insect may become scared when it was fixed into the magnet, leading to the release of some chemical material in its body and thus causing spectral changes in its bioluminescence. The second one is that the insect's nervous system may be affected by the external magnetic field and causes changes (e.g., $\mathrm{pH})$ to the in vivo reaction environment, and thus causing bioluminescent spectral changes. These two factors are possible as firefly bioluminescence is well-known to be very sensitive to the reaction environment. And thirdly, the applied magnetic field may induce changes to the luciferin-luciferase chemical reaction, such as the balance between cage and escape products and the yield of certain intermediate molecules. Finally, another possibility is that the magnetic field used by Iwasaka et al could have affected the intermolecular interactions (as hydrogen bonding, electrostatic, $\pi-\pi$ stacking) between oxyluciferin and the active site of luciferase [38-42] and thus causing spectral changes. 
Nonetheless, what we could conclude from our data is, that the spectral changes observed by Iwasaka et $a l$., if true, are related neither to the light emitter oxyluciferin nor to its intrinsic energy states.

Besides the magnetic field induced spectral changes, another important but yet unresolved issue in firefly bioluminescence is its environment-sensitive emission colors [5, 20-21, 23, 43-46]. Numerous in vitro experiments have shown that emission color of firefly bioluminescence can be changed very sensitively between yellow-green and red by modifications to the reaction environment, such as $\mathrm{pH}$, temperature and bivalent metal ion additives [5, 9, 47-48]. Although several mechanisms have been proposed to account for this peculiar environment-sensitive emission color changes, no consensus has ever been reached so far. In our previous work, we revealed, using quantitative spectroscopy, that firefly bioluminescence spectra can be systematically decomposed into three Gaussian components $[5,34]$. What is interesting is that we found the green component at $\sim 2.2 \mathrm{eV}$ is a special one. Our data show that only the green component at $\sim 2.2 \mathrm{eV}$ is sensitive to the reaction environment while the orange and red components do not. Moreover, the simple intensity variation of this green component accounts for the entire emission color changes, calling the proposed color change mechanism based on chemical equilibrium [43-44] into question. However in this work, we demonstrate that the green component exhibits the same behavior as the orange and red components when subjected to strong magnetic field, i.e., all of them are robust against the external field. Although we are still unable to specify the exact mechanism for the environment-sensitive emission colors at this moment, the magnetic field independent phenomenon we observed in this work does serve as an exception to our previous findings using quantitative spectroscopy. This may help theorists develop more accurate models to account for the mysterious environment-sensitive emission colors of firefly bioluminescence.

Finally, we would like to point out that the possible byproduct, dehydroluciferin which 
presents similar optical properties with oxyluciferin [49-51], would not cause any confusion to our conclusion. Our main finding is that emission from the luciferin-luciferase reaction product remains unchanged in high magnetic fields. This undoubtedly leads to our conclusion, that emission properties of the oxyluciferin, and actually other possible byproducts as well, are immune to external high magnetic fields, regardless of the possible existence of other byproducts.

\section{Conclusions}

In summary, we performed in situ magneto-PL study on firefly light emitter oxyluciferin under very high magnetic fields up to $53 \mathrm{~T}$. In contrary to the previous report showing significant magnetic field effect on firefly bioluminescence, we confirmed, by means of selective excitation, that emission properties of firefly light emitter oxyluciferin are immune to external high magnetic fields up to $53 \mathrm{~T}$. Meanwhile, the green Gaussian component at $\sim 2.2 \mathrm{eV}$ exhibits the same behavior as the orange and red Gaussian components when subjected to high magnetic field, in strong contrast to previous findings showing distinctive behaviors for the green component. As oxyluciferin is the real light emitting molecule in firefly bioluminescence, the work reported here could help clarify the mechanism of firefly emission color changes and facilitate their future applications.

\section{Acknowledgments}

We thank Professor K. Kindo for supplying us a nondestructive pulsed magnet. W. H. Zhou thanks for the financial support of the post-doctoral research fellowship at the Institute for Solid State Physics, University of Tokyo. Financial supports from KAKENHI No.26610081 (JSPS) and JST-SENTAN are also gratefully acknowledged. 


\section{References}

[1] C. B. Grissom, Magnetic Field Effects in Biology: A Survey of Possible Mechanisms with Emphasis on Radical-Pair Recombination, Chem. Rev. 95 (1995) 3

[2] T. T. Harkins, C. B. Grissom, Magnetic Field Effects on $B_{12}$ Ethanolamine Ammonia Lyase: Evidence for a Radical Mechanism, Science 263 (1994) 958

[3] W. Haberditzl, Enzyme Activity in High Magnetic Fields, Nature 213 (1967) 72

[4] M. Iwasaka, S. Ueno, Bioluminescence under static magnetic fields, J. Appl. Phys. 83 (1998) 6456

[5] Y. Ando, K. Niwa, N. Yamada, T. Enomoto, T. Irie, H. Kubota, Y. Ohmiya, H. Akiyama, Firefly bioluminescence quantum yield and colour change by $\mathrm{pH}$-sensitive green emission, Nat. Photonics 2 (2007) 44

[6] S. M. Lewis, C. K. Cratsley, Flash Signal Evolution, Mate Choice, and Predation in Fireflies, Annu. Rev. Entomol. 53 (2008) 293

[7] O. Shimomura, Bioluminescence: Chemical Principles and Methods, World Scientific, New Jersey, 2006, pp.1-29

[8] H. H. Seliger, W. D. McElroy, Quantum yield in the oxidation of firefly luciferin, Biochem. Biophys. Res. Commun. 1 (1959) 21

[9] H. H. Seliger, W. D. McElroy, Spectral emission and quantum yield of firefly bioluminescence, Arch. Biochem. Biophys. 88 (1960) 136

[10]Luis Pinto da Silva, R. Simkovitch, D. Huppert, Joaquim C.G. Esteves da Silva, Oxyluciferin Photoacidity: The Missing Element for Solving the Keto-Enol Mystery?, ChemPhysChem 14 (2013) 3441

[11]S. Hosseinkhani, Molecular enigma of multicolor bioluminescence of firefly luciferase, Cell. Mol. Life. Sci. 68 (2011) 1167

[12]A. Lundin, Optimization of the firefly luciferase reaction for analytical purposes, Adv. Biochem. Eng. Biotechnol. 145 (2014) 31 
[13]J. Vieira, Luis Pinto da Silva, Joaquim C.G. Esteves da Silva, Advances in the knowledge of light emission by firefly luciferin and oxyluciferin, J. Photochem. Photobiol. B 117 (2012) 33

[14]Luis Pinto da Silva, Joaquim C.G. Esteves da Silva, Firefly Chemiluminescence and Bioluminescence Efficient Generation of Excited States, ChemPhysChem 13 (2012) 2257

[15]S. Inouye, Firefly luciferase: an adenylate-forming enzyme for multicatalytic functions, Cell. Mol. Life. Sci. 67 (2010) 387

[16]H. Fraga, Joaquim C. G. Esteves da Silva, R. Fontes, Identification of Luciferyl Adenylate and Luciferyl Coenzyme A Synthesized by Firefly Luciferase, ChemBioChem 5 (2004) 110

[17]Luis Pinto da Silva, Joaquim C.G. Esteves da Silva, Theoretical modulation of the color of light emitted by firefly oxyluciferin, J. Comput. Chem. 32 (2011) 2654

[18]R. Fontes, D. Fernandes, F. Peralta, H. Fraga, I. Maio, Joaquim C. G. Esteves da Silva, Pyrophosphate and tripolyphosphate affect firefly luciferase luminescence because they act as substrates and not as allosteric effectors, FEBS Journal 275 (2008) 1500

[19] Y. Erez, I. Presiado, R. Gepshtein, Luis Pinto da Silva, Joaquim C. G. Esteves da Silva, D. Huppert, Comparative Study of the Photoprotolytic Reactions of d-Luciferin and Oxyluciferin, J. Phys. Chem. A 116 (2012) 7452

[20]P. Naumov, Y. Ozawa, K. Ohkubo, S. Fukuzumi, Structure and Spectroscopy of Oxyluciferin, the Light Emitter of the Firefly Bioluminescence, J. Am. Chem. Soc. $131(2009) 11590$

[21]T. Hirano, Y. Hasumi, K. Ohtsuka, S. Maki, H. Niwa, M. Yamaji, D. Hashizume, Spectroscopic Studies of the Light-Color Modulation Mechanism of Firefly (Beetle) Bioluminescence, J. Am. Chem. Soc. 131 (2009) 2385

[22]N. Nakatani, J. Hasegawa, H. Nakatsuji, Red Light in Chemiluminescence and 
Yellow-Green Light in Bioluminescence: Color-Tuning Mechanism of Firefly, Photinus pyralis, Studied by the Symmetry-Adapted Cluster-Configuration Interaction Method, J. Am. Chem. Soc. 129 (2007) 8756

[23]T. Nakatsu, S. Ichiyama, J. Hiratake, A. Saldanha, N. Kobashi, K. Sakata, H. Kato, Structural basis for the spectral difference in luciferase bioluminescence, Nature 440 (2006) 372

[24]H. H. Seliger, W. D. McElroy, E. H. White, G. F. Field, Stereospecificity and firefly bioluminescence, a comparison of natural and synthetic luciferins, Proc. Natl. Acad. Sci. USA 47 (1961) 1129

[25]E. H. White, F. McCapra, G. F. Field, W. D. McElroy, The structure and synthesis of firefly luciferin, J. Am. Chem. Soc. 83 (1961) 2402

[26]E. H. White, F. McCapra, G. F. Field, The Structure and Synthesis of Firefly Luciferin, J. Am. Chem. Soc. 85 (1963) 337

[27]Luis Pinto da Silva, Joaquim C. G. Esteves da Silva, Chemiexcitation Induced Proton Transfer: Enolate Oxyluciferin as the Firefly Bioluminophore, J. Phys. Chem. B 119 (2015) 2140

[28]K. M. Solntsev, S. P. Laptenok, P. Naumov, Photoinduced Dynamics of Oxyluciferin Analogues: Unusual Enol "Super" photoacidity and Evidence for Keto-Enol Isomerization, J. Am. Chem. Soc. 134 (2012) 16452

[29]Luis Pinto da Silva, A. Joel M. Santos, Joaquim C. G. Esteves da Silva, Efficient Firefly Chemi/Bioluminescence: Evidence for Chemiexcitation Resulting from the Decomposition of a Neutral Firefly Dioxetanone, J. Phys. Chem. A 117 (2013) 94

[30]Joao M. M. Leitao, Joaquim C. G. Esteves da Silva, Firefly luciferase inhibition, J. Photochem. Photobiol. B 101 (2010) 1

[31]H. Isobe, Y. Takano, M. Okumura, S. Kuramitsu, K. Yamaguchi, Mechanistic Insights in Charge-Transfer-Induced Luminescence of 1,2-Dioxetanones with a Substituent of 
Low Oxidation Potential, J. Am. Chem. Soc. 127 (2005) 8667

[32]L. Yue, Ya-Jun Liu, Wei-Hai Fang, Mechanistic Insight into the Chemiluminescent Decomposition of Firefly Dioxetanone, J. Am. Chem. Soc. 134 (2012) 11632

[33]Luis Pinto da Silva, Joaquim C. G. Esteves da Silva, Interstate Crossing-Induced

Chemiexcitation as the Reason for the Chemiluminescence of Dioxetanones, ChemPhysChem 14 (2013) 1071

[34] Y. Wang, H. Akiyama, K. Terakado, T. Nakatsu, Impact of Site-Directed Mutant Luciferase on Quantitative Green and Orange/Red Emission Intensities in Firefly Bioluminescence, Sci. Rep. 3 (2013) 2490

[35] Y. Wang, Y. Hayamizu, H. Akiyama, Spectroscopic Study of Firefly Oxyluciferin in an Enzymatic Environment on the Basis of Stability Monitoring, J. Phys. Chem. B 118 (2014) 2070

[36]W. Zhou, T. Sasaki, D. Nakamura, H. Saito, H. Liu, H. Kataura, S. Takeyama, Survey of exciton-phonon sidebands by magneto-optical spectroscopy using highly specified (6,5) single-walled carbon nanotubes, Appl. Phys. Lett. 103 (2013) 021117

[37]W. Zhou, T. Sasaki, D. Nakamura, H. Saito, H. Liu, H. Kataura, S. Takeyama, Exciton-phonon bound complex in single-walled carbon nanotubes revealed by high-field magneto-optical spectroscopy, Appl. Phys. Lett. 103 (2013) 233101

[38]Luis Pinto da Silva, Joaquim C. G. Esteves da Silva, TD-DFT/Molecular Mechanics Study of the Photinus pyralis Bioluminescence System, J. Phys. Chem. B 116 (2012) 2008

[39]I. Navizet, Ya-Jun Liu, N. Ferre, Hong-Yan Xiao, Wei-Hai Fang, R. Lindh, Color-Tuning Mechanism of Firefly Investigated by Multi-Configurational Perturbation Method, J. Am. Chem. Soc. 132 (2010) 706

[40]Bruce F. Milne, Miguel A. L. Marques, F. Nogueira, Fragment molecular orbital investigation of the role of AMP protonation in firefly luciferase $\mathrm{pH}$-sensitivity, Phys. 
Chem. Chem. Phys. 12 (2010) 14285

[41]Luis Pinto da Silva, Joaquim C. G. Esteves da Silva, Study on the Effects of Intermolecular Interactions on Firefly Multicolor Bioluminescence, ChemPhysChem $12(2011) 3002$

[42]Luis Pinto da Silva, R. Simkovitch, D. Huppert, Joaquim C. G. Esteves da Silva, Theoretical Photodynamic Study of the Photoprotolytic Cycle of Firefly Oxyluciferin, ChemPhysChem 14 (2013) 2711

[43]E. H. White, E. Rapaport, T. A. Hopkins, H. H. Seliger, Chemi- and bioluminescence of firefly luciferin, J. Am. Chem. Soc. 91 (1969) 2178

[44]E. H. White, E. Rapaport, H. H. Seliger, T. A. Hopkins, The Chemi- and Bioluminescence of Firefly Luciferin: An Efficient Chemical Production of Electronically Excited States, Bioorg. Chem. 1 (1971) 92

[45]B. R. Branchini, T. L. Southworth, M. H. Murtiashaw, R. A. Magyar, S. A. Gonzalez, M. C. Ruggiero, J. G. Stroh, An Alternative Mechanism of Bioluminescence Color Determination in Firefly Luciferase, Biochemistry 43 (2004) 7255

[46]N. N. Ugarova, L. G. Maloshenok, I. V. Uporov, M. I. Koksharov, Bioluminescence spectra of native and mutant firefly luciferases as a function of $\mathrm{pH}$, Biochemistry 70 (2005) 1262

[47]H. H. Seliger, W. D. McElroy, The colors of firefly bioluminescence: enzyme configuration and species specificity, Proc. Natl. Acad. Sci. USA 52 (1964) 75

[48]Y. Wang, H. Kubota, N. Yamada, T. Irie, H. Akiyama, Quantum Yields and Quantitative Spectra of Firefly Bioluminescence with Various Bivalent Metal Ions, Photochem. Photobiol. 87 (2011) 846

[49]H. Fraga, D. Fernandes, J. Novotny, R. Fontes, Joaquim C. G. Esteves da Silva, Firefly Luciferase Produces Hydrogen Peroxide as a Coproduct in Dehydroluciferyl Adenylate Formation, ChemBioChem 7 (2006) 929 
[50]Luis Pinto da Silva, Joaquim C. G. Esteves da Silva, Kinetics of inhibition of firefly luciferase by dehydroluciferyl-coenzyme A, dehydroluciferin and L-luciferin, Photochem. Photobiol. Sci. 10 (2011) 1039

[51]I. Presiado, Y. Erez, R. Simkovitch, S. Shomer, R. Gepshtein, Luis Pinto da Silva, Joaquim C.G. Esteves da Silva, D. Huppert, Excited-State Proton Transfer of Firefly Dehydroluciferin, J. Phys. Chem. A 116 (2012) 10770 


\section{Figure Captions}

Fig. 1. Reaction mechanism of the luciferase-catalyzed firefly bioluminescence summarized into three steps. The final reaction product oxyluciferin has six possible forms and presented here in its neutral keto form. Symbol "*" in Step II \& III represent electronically excited state of oxyluciferin.

Fig. 2. (a) Spectrum of Luciola cruciata firefly bioluminescence at room temperature. Together shown in the figure are the three Gaussian components that reproduce the spectrum. Green dash-dotted line: $\mathrm{pH}$-sensitive green component at $~ 2.2 \mathrm{eV}$ (Peak "G"); Orange dash-dot-dotted and red dashed lines: $\mathrm{pH}$-insensitive orange and red components at $2.0 \mathrm{eV}($ Peak "O") and $1.88 \mathrm{eV}$ (Peak "R"), respectively. $\mathrm{pH}$ of the reaction solution: 6.6. (b) In situ photoluminescence of the light emitter oxyluciferin $\left(\mathrm{OxyLH}_{2}\right)$ in complex with luciferase. Green line: photoluminescence with excitation at $430 \mathrm{~nm}$ (selected from a halogen lamp by monochromator); Pink line: photoluminescence excited by laser at 532 $\mathrm{nm}$; Green dashed line: excitation light at $430 \mathrm{~nm}$; pink dashed line: excitation light at 532 nm.

Fig. 3. Photoluminescence of oxyluciferin under high magnetic field and with laser excitation at $406 \mathrm{~nm}$.

Fig. 4. Photoluminescence of oxyluciferin under high magnetic field and with laser excitation at $532 \mathrm{~nm}$. 
Figures

(I)

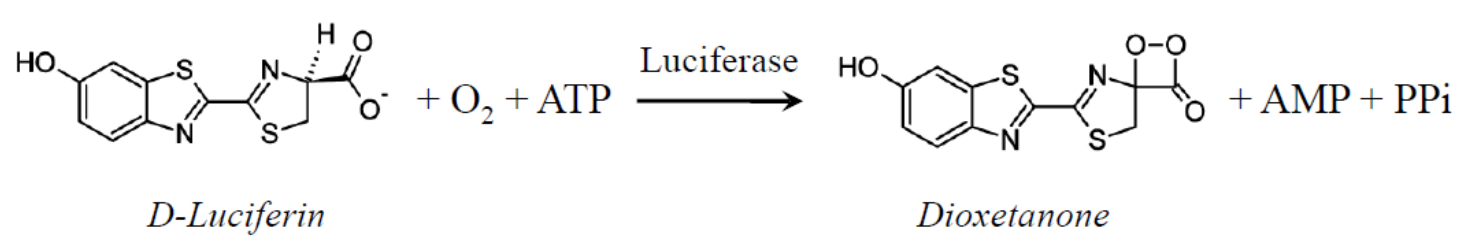

(II)

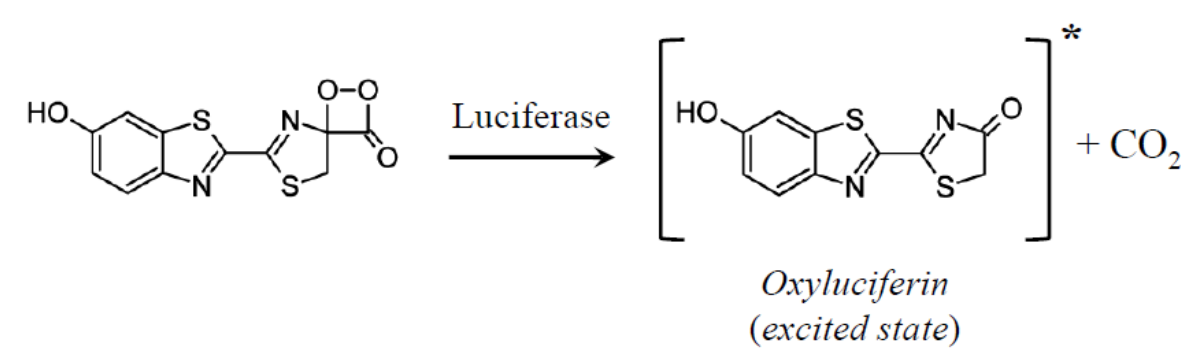

(III)

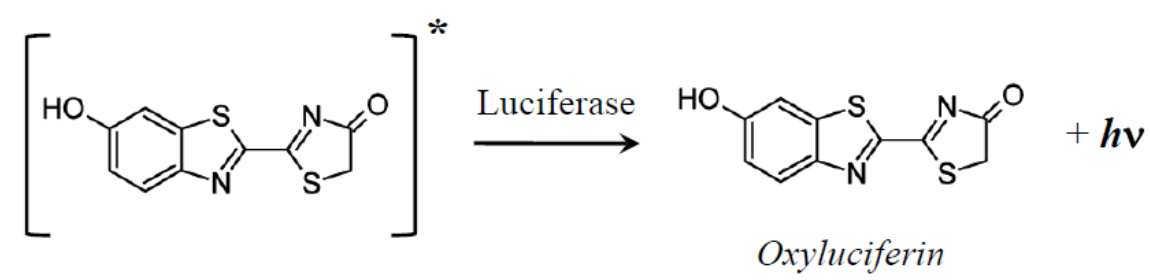

Fig.1. 

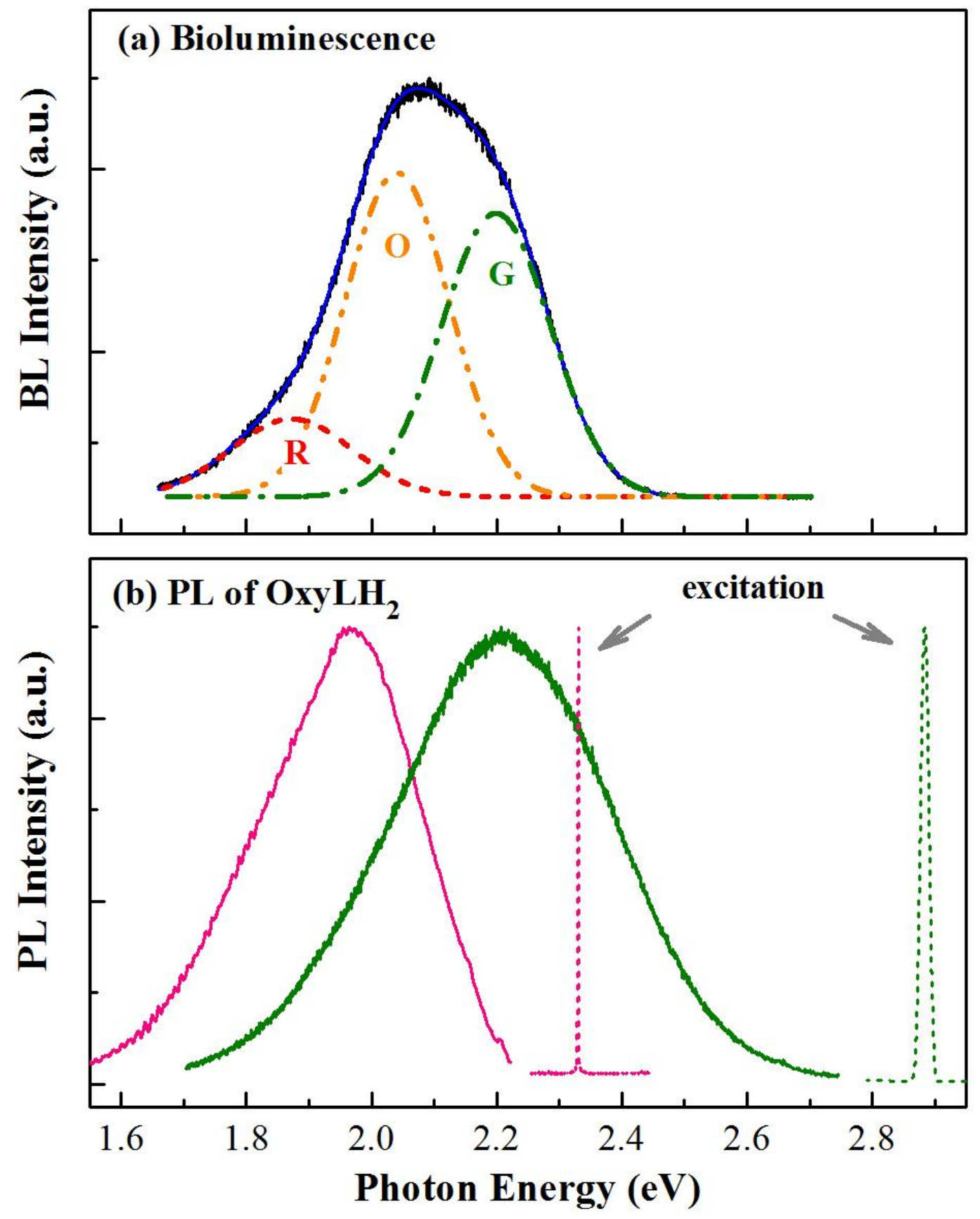

Fig. 2. 


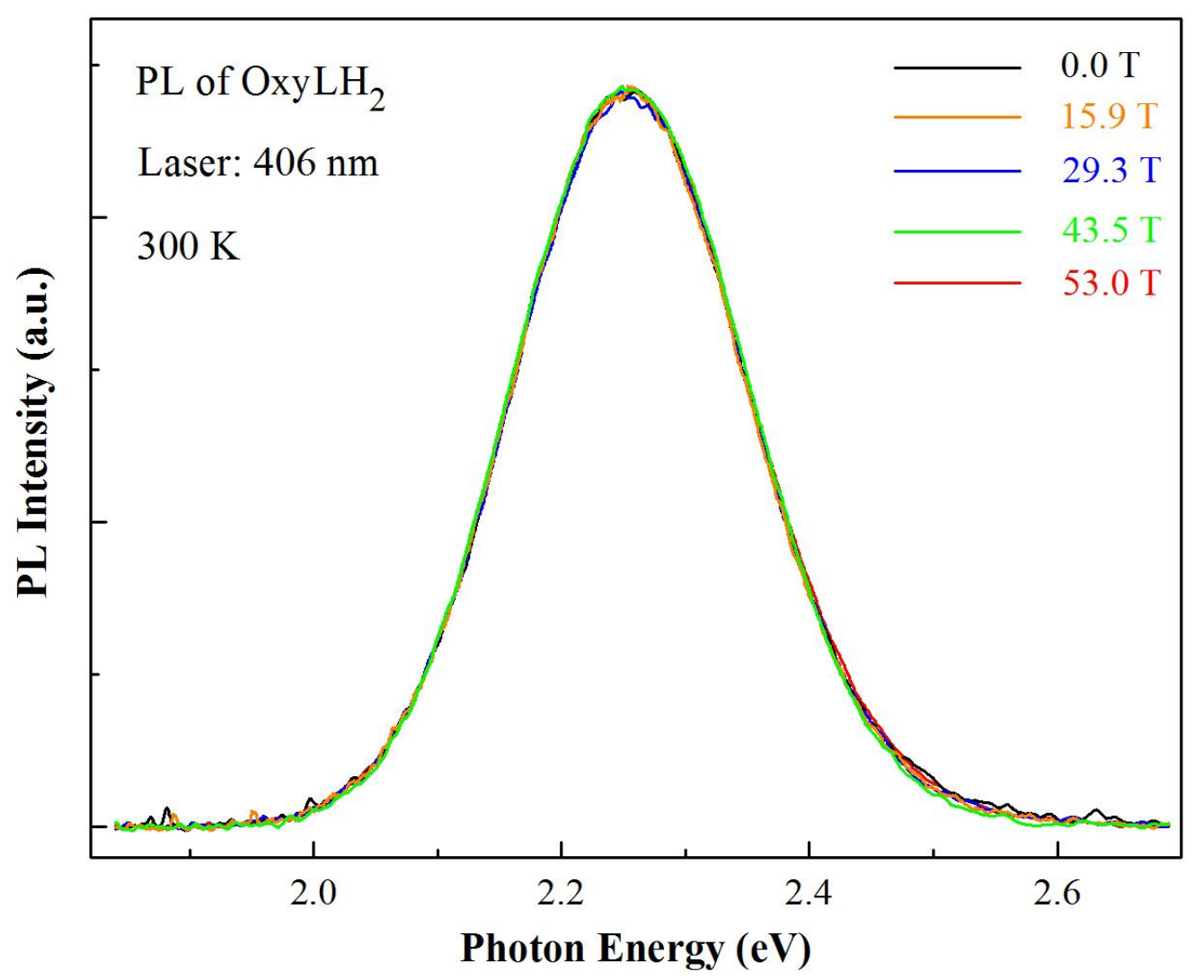

Fig. 3. 


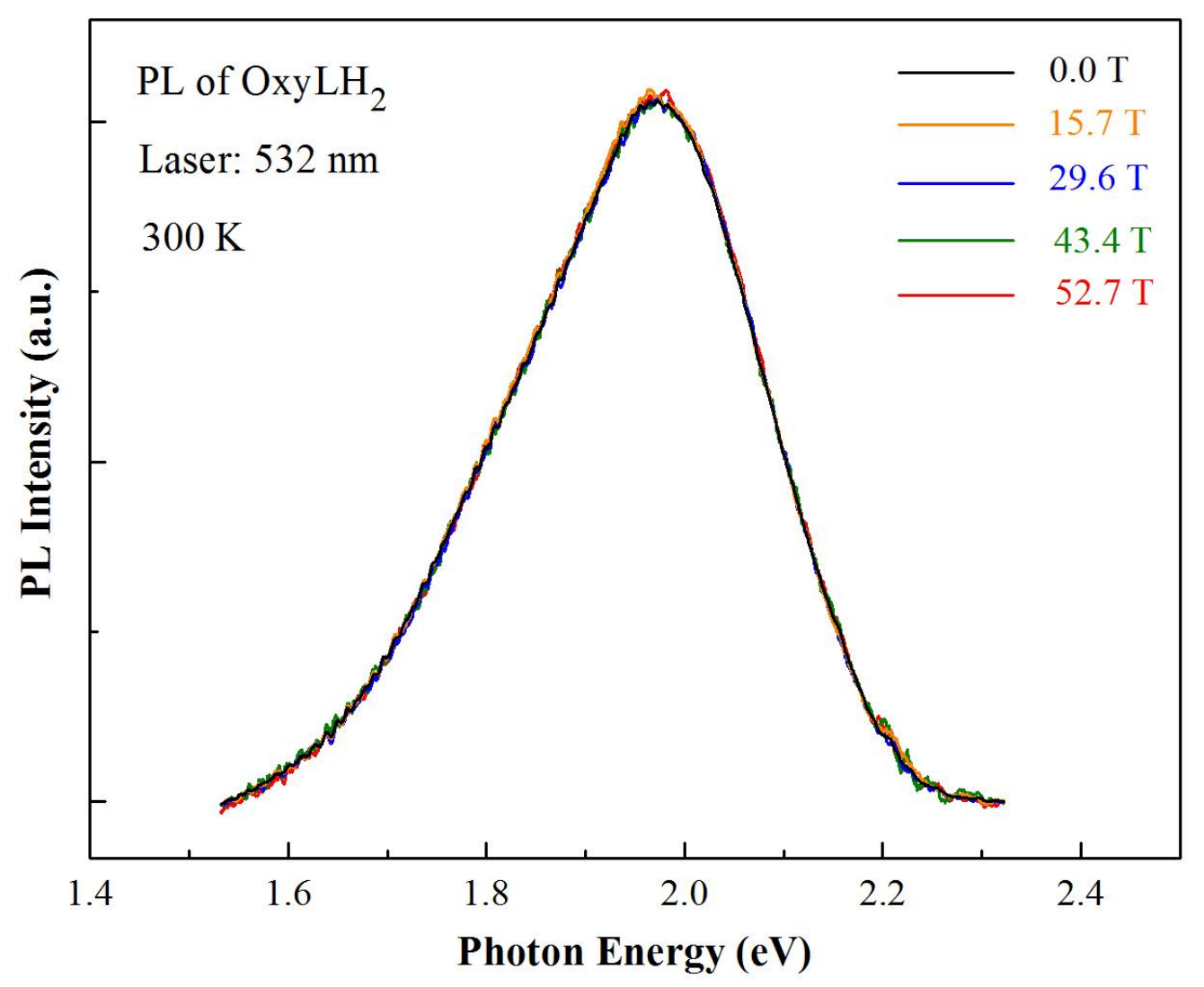

Fig. 4. 